\title{
Stereological Analysis of the Statistical Distribution of the Size of Graphite Nodules in DI
}

\author{
Andriy Burbelko ${ }^{a *}$, Daniel Gurgul ${ }^{b}$, Edward Guzik ${ }^{c}$ \\ and Wojciech Kapturkiewicz ${ }^{\mathrm{d}}$
}

\author{
AGH University of Science and Technology, Mickiewicza Av. 30, 30-059 Krakow, Poland \\ aabur@agh.edu.pl, bdg@agh.edu.pl, cguz@agh.edu.pl, dkaptur@agh.edu.pl
}

Keywords: graphite nodules, ductile iron, graphite size distribution, chord length, stereological analysis

\begin{abstract}
The estimate of a distribution law of the nodule diameters in a volume of cast iron provides information about the graphite nucleation kinetics, and also about the crystallization kinetics. This information is essential for building more accurate mathematical models of the alloy crystallization. The mapping of a Cumulative Distribution Function $\left(\mathrm{CDF}_{3}\right)$ of radii for graphite nodules in ductile iron is presented on the base of a Probability Density Function $\left(\mathrm{PDF}_{1}\right)$ of the chord length distribution for random sections of the sample at the planar cross-section.
\end{abstract}

\section{Introduction}

Ductile Cast Iron (DI) is a widely used alloy of the modern technique due to his high level of functional and technological quality. Depending on the chemical composition and processing method, the mechanical properties of DI can vary within a wide range. Among the factors that influence on the properties of DI are the volume fraction of the graphite nodules, their volumetric quantity and size distribution.

In the paper of Yin at al. [1] the results of the use of X-ray microtomography for estimation a three-dimensional size distribution and quantitative characteristic of the morphology of graphite nodules in the stepwise casting of cast iron from cast iron are presented. Unfortunately, the graphite nodules whose diameter is smaller than $25 \mu \mathrm{m}$ are ignored because of the resolution of $3 \mathrm{D}$ volume and the interference of inclusion and microporosity. Probably, it is for this reason that samples of castings with a wall thickness of less than $15 \mathrm{~mm}$ were not analyzed.

The stereological analysis of the 2D image is used most often for the analysis of the characterization of the properties of the set of spherical particles in the nontransparent material like DI $[2,3]$.

The determination of probability density function $f_{3}\left(r_{3}\right)$ of the radii of graphite nodules $\left(\mathrm{PDF}_{3}\right)$ occurring in a volume of ductile cast iron using data on the probability density function of the radii of the cross-sections of these nodules $f_{2}\left(r_{2}\right)$ in a metallographic specimen $\left(\mathrm{PDF}_{2}\right)$ can be regarded as a special case of Wicksell's corpuscle problem (WCP) [4]. According to Wicksell $\mathrm{PDF}_{2}$ can be calculated explicitly based on $\mathrm{PDF}_{3}$ as follow:

$$
f_{2}(t)=\frac{t}{E\left[r_{3}\right]} \int_{t}^{R_{\max }} \frac{f_{3}(x)}{\sqrt{x^{2}-t^{2}}} \mathrm{~d} x,
$$

where $R_{\max }$ denotes the maximum radius of the nodules in the sample, and $E\left[r_{3}\right]$ is the expected (mean) value of the radius of spherical particles $r_{3}$ in this sample.

For the estimation of the $\mathrm{PDF}_{3}$ based on $\mathrm{PDF}_{2}$ the inverse solution of the Eq. (1) should be obtained. In practical metallographic tasks, the application of analytical solutions to WCP on the base of $\mathrm{PDF}_{2}$ gives unsatisfactory results [3], therefore there is a need for the use of implicit numerical solution method of Eq. (1).

Application of the Wicksell equation for the estimation of the size distribution of spherical particles on the base of empirical distribution of the sizes of planar sections has been presented by Sheil [5], Schwartz [6], Saltykov [7,8], Li at al. [9]. A similar solution for the mineralogy task has 
been presented in [10]. Unfortunately, small numerous errors of the empirically estimated function $f_{2}(t)$ result in the ,arbitrarily large perturbations of the solution” $[11,12]$.

\section{Research Method}

Theoretical base. As it is has been proved in [13] the empirical cumulative distribution function of the spherical particles size $\left(\mathrm{CDF}_{3}\right)$ can be calculated on the basis of the statistical distribution of the length of the chords $\left(\mathrm{PDF}_{1}\right)$ obtained by intersecting spherical particles with a system of random secants. $\mathrm{CDF}_{3}$ can be mapped by equation:

$$
F_{3}(t)=1-\frac{\bar{S}}{8 \pi t} f_{1}(t)
$$

where $\bar{S}$ is the mean external surface (estimated value) of the nodular particles in the sample, and $f_{1}(t)$ is the $\mathrm{PDF}_{1}$.

The derivations of Eq. (2) with respect $t$ parameter gives:

$$
f_{3}(t)=\frac{\bar{S}}{8 \pi}\left[\frac{f_{1}(t)}{t^{2}}-\frac{1}{t} \frac{\mathrm{d} f_{1}(t)}{\mathrm{d} t}\right] \text {. }
$$

This formulation corresponds to known solutions of Cahn and Fullmann [14], Lord and Willis [15], and Spektor [16]:

$$
N_{3} f_{3}(t)=\frac{N_{1}}{2 \pi}\left[\frac{f_{1}(t)}{t^{2}}-\frac{1}{t} \frac{\mathrm{d} f_{1}(t)}{\mathrm{d} t}\right] .
$$

Verification of the method. Correctness of the Eq. (2) application has been verified in [17]. Two sets of virtual spherical particles with inhomogeneous dimensions were generated: normal distribution (2048 particles with mean radius 120 units and standard deviation 15 units) and bimodal one as the superposition of two normal distributions (2048 particles with mean radius / std. deviation 90 / 10 and 1024 particles 150 /150). Comparison of the mapping results of the $\mathrm{CDF}_{3}$ with the theoretical values for above distributions is presented in Fig. 1.

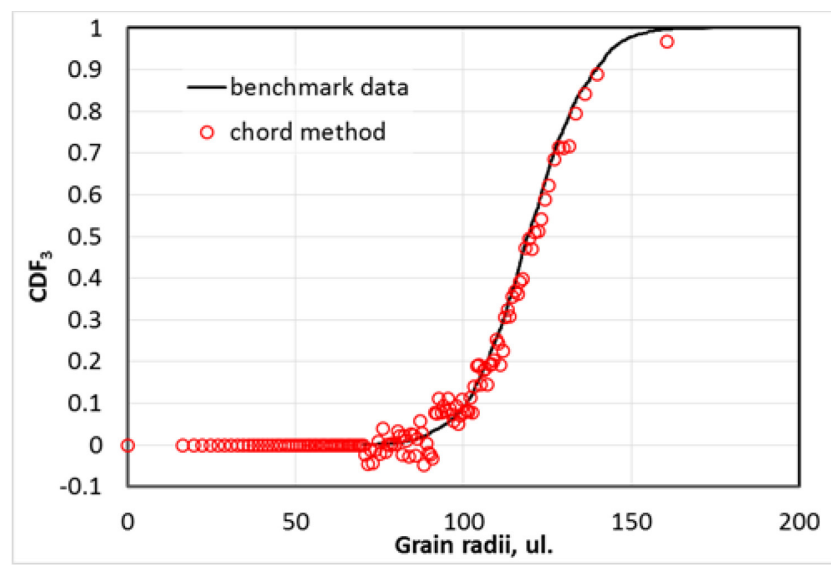

a)

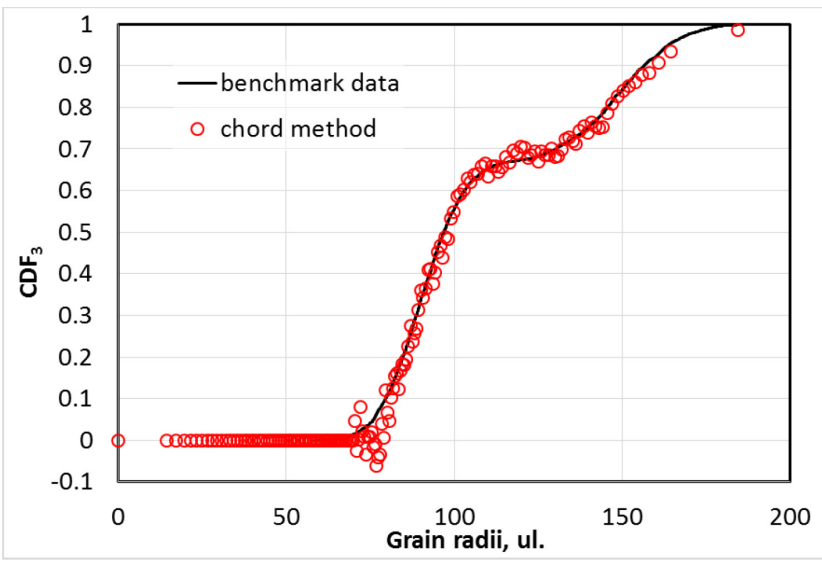

b)

Fig. 1. $\mathrm{CDF}_{3}$ of the radii of the spherical particles obtained directly on the base of the normal distribution properties (solid lines) and results of the Eq. (2) application (circles): a) normal distribution; b) bimodal distribution [17] (ul. means the unit length).

\section{Sample Preparation and Data Acquisition}

Analyzed DI. As the subject of the measurements the samples were using from the industrial casting produced from DI. The chemical composition of the iron was measured by spectrometer 
LECO type GDS500A and apparatus HORIBA Scientific type EMIA $820 \mathrm{~B}(\mathrm{~W})$ for carbon and sulfur. Chemical composition of the iron used for casting production is presented in the Table 1 .

Table 1. Chemical composition of the DI [mass \%].

\begin{tabular}{|c|c|c|c|c|c|c|c|c|c|c|}
\hline $\mathrm{C}$ & $\mathrm{Si}$ & $\mathrm{Mn}$ & $\mathrm{S}$ & $\mathrm{P}$ & $\mathrm{Cu}$ & $\mathrm{Cr}$ & $\mathrm{Mg}$ & $\mathrm{Ni}$ & $\mathrm{Ti}$ & $S_{c}$ \\
\hline 3.61 & 2.53 & 0.35 & 0.009 & 0.02 & 0.19 & 0.01 & 0.045 & 0.01 & 0.01 & 1.04 \\
\hline
\end{tabular}

where eutectic saturation ratio $S_{c}=\mathrm{C} /(4.26-0.3 \cdot \mathrm{Si}-0.36 \cdot \mathrm{P})$.

Sample preparation. Two metallographic specimen for stereological measurements have been fetched from the different casting sections: No. I and No. II, with different wall thickness and with different cooling rate. The sections for analysis were subjected to multiple polishing and etching with Nital to remove the deformed layer and minimize the relief. In the microstructure of the samples the precipitations of nodular graphite of the type VI (in accordance with the standard PNISO 945-1:2009) in the amount $80 \%$ minimum have been revealed (remainder form the irregular V graphite and vermicular graphite shapes). The measurements and statistical analysis were performed for graphite nodules of the type VI.

Microstructure of the samples etched by Nital is presented in Fig. 2. Metallic matrix of the specimen No. I consists of $54.3 \%$ vol. of ferrite an $39.8 \%$ of pearlite, while in the matrix of specimen No. II volume fraction of ferrite is $35.3 \%$ and of pearlite $-56.9 \%$.

Data acquisition. Data on the statistical distribution of the length of the random chords in the sections of graphite spheroids were collected as a result of measurements on un-etched metallographic sections. The examples of the microstructure of the un-etched sections used for data acquisition are presented in Fig. 3. Sets of the measurements results were acquired on a metallographic microscope Leica MEF4M. For each sample, 50 photos were taken into account at magnification x100 (2048 x 1536 pix, resolution $0.479 \mu \mathrm{m} /$ pix $)$.

Preliminary data processing by using the Leica QWin software. Each analyzed image of a microstructure was transformed into a binary image. The binary images included only these areas from the original image which could be potentially taken for measurements. All pixels belonging to the matrix were discarded. Having the binary images it was necessary to clean them from the areas which were unsuitable for the analysis. In this work all detected cohesive group of pixels with the area less than 15 pixels $\left(3.5 \mu \mathrm{m}^{2}\right)$ were rejected from the data set as noise. Also, if the shape of each group was significantly different from the circular one, this group was deleted. Determination of the shape was done by using two factors from the QWin software i.e. AspectRatio and Roundness. For both factor a limit was set from 1 to 1.2. Each group which did not belong to this limit was not taken into consideration. In addition, compact pixel groups were not included in the measurements if their geometric center was located at a distance less than the radius of the largest cross section of particles from the image boundary.

\section{Obtained Results and Discussion}

Data processing. The total length of the secants used for measuring the length of the chords was $49,280,000$ pix $(23,593,046 \mu \mathrm{m})$. The calculations using the Eq. (2)were performed on the data for $66862 \mathrm{D}$ circular cross-sections of the graphite nodules of the type VI and 176507 chords for sample No. I, and 6745 2D circular cross-sections and 162556 chords for sample No. II.

The average surface area of the nodules was calculated by dividing the total surface area of all particles per unit volume $\left(S_{V}\right)$ by the volumetric number of particles (grain density $N_{V}$ ):

$$
\bar{S}=S_{V} / N_{V} \text {. }
$$




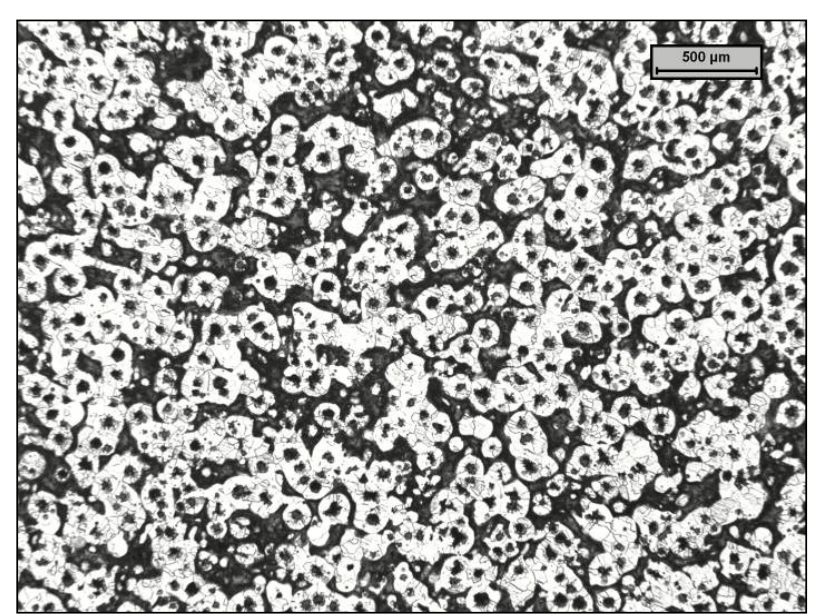

a)

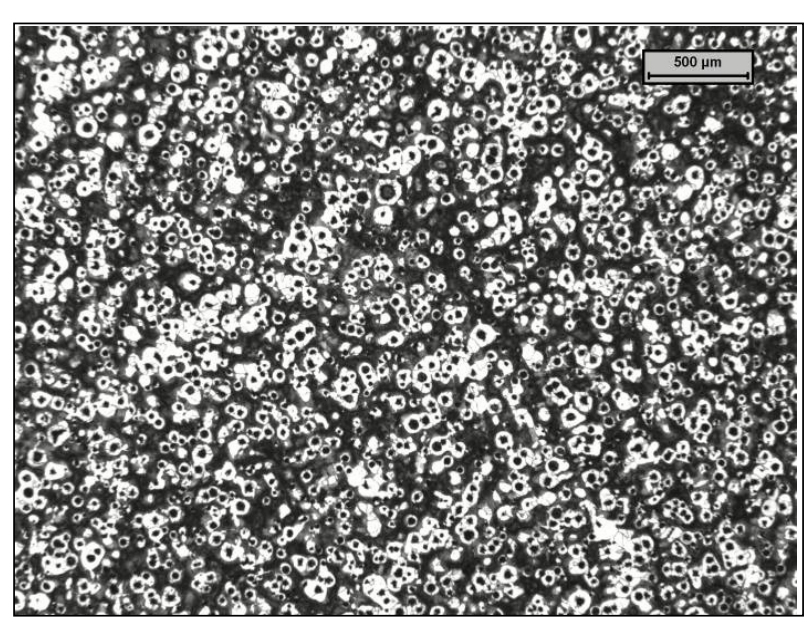

b)

Fig. 2. Microstructure of the samples of DI, etched by Nital, x25: a) probe No. I, b) probe No. II.

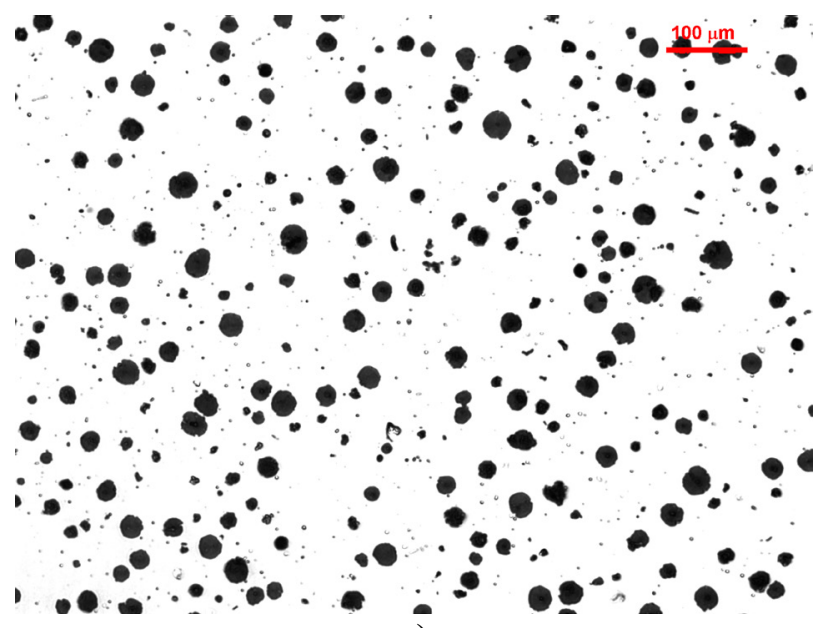

a)

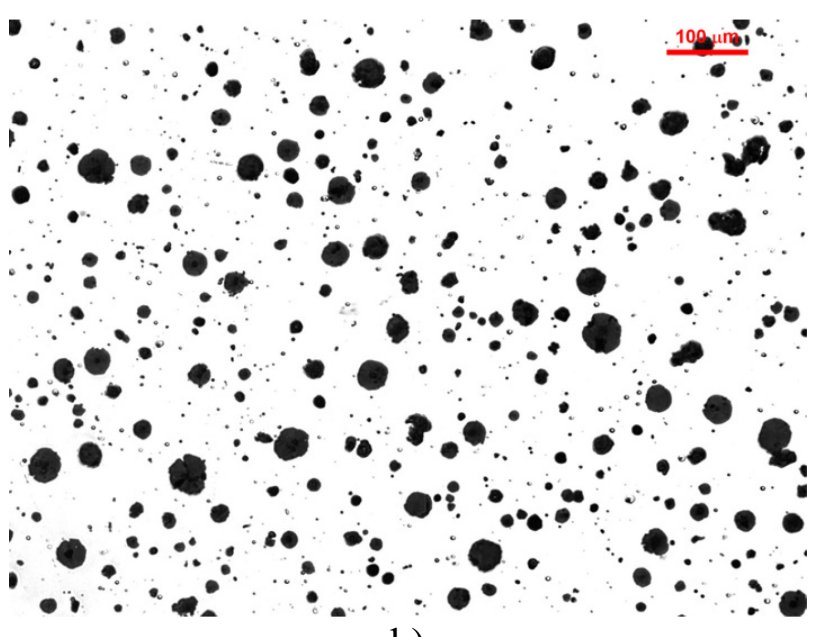

b)

Fig. 3. Microstructure of the samples of DI, un-etched, x100: a) probe No. I, b) probe No. II.

These parameters for Eq. (5) were calculated by the Saltykov's secant method for $S_{V}$

$$
S_{V}=2 \cdot P_{L},
$$

and by the Saltykov's method of invers diameter for $N_{V}$

$$
N_{V}=\frac{2}{\pi F} \sum_{i} \frac{1}{d_{i}}
$$

where: $P_{L}$ - the number of intersections of the surface of graphite nodules per unit length of the secants, $F$ - total surface area of the analyzed metallographic sections, $d_{i}$ - diameters of the 2D sections $i$.

The results of the average surface area estimation for the graphite nodules are presented in the Table 2. The empirical cumulative distribution functions and probability density function prepared on the base of measurement are presented in Fig. 4.

Table 2. Averaged surface area of the graphite nodules in the DI samples.

\begin{tabular}{|c|c|c|c|}
\hline Sample & $S_{V}\left[\mu \mathrm{m}^{2} / \mu \mathrm{m}^{3}\right]$ & $N_{V}\left[1 / \mu \mathrm{m}^{3}\right]$ & $\bar{S}\left[\mu \mathrm{m}^{2}\right]$ \\
\hline No. I & 14.7 & 7930 & 1850 \\
\hline No. II & 13.8 & 9790 & 1410 \\
\hline
\end{tabular}




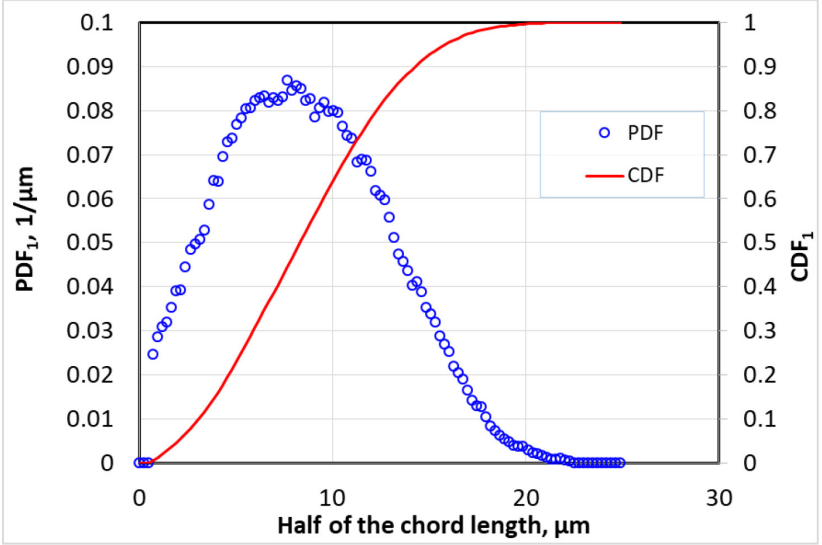

a)

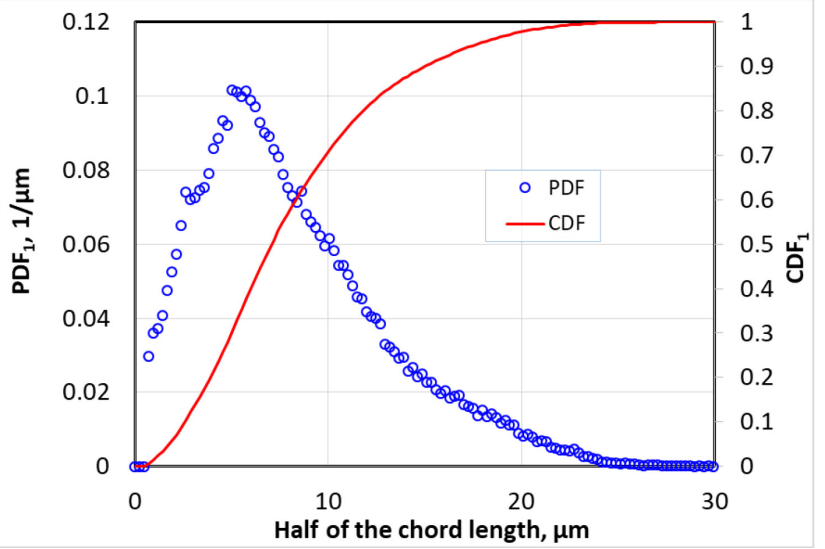

b)

Fig. 4. Empirical cumulative distribution function (CDF) and probability density function (PDF) of the chord length: a) probe No. I, b) probe No. II.

Distribution of the sizes of graphite nodules. Results of mapping of graphite nodules sizes calculated by Eq. 2 are presented in Fig. 5a. As follows from this diagram, the chord method has a low accuracy for the smallest $10 \%$ of the spheroids in the analyzed population. For the samples described, this interval includes spherical particles with a radius of less than about $7 \mu \mathrm{m}$. Unfortunately, for $r_{3}<6 \mu \mathrm{m}$ the result of $\mathrm{CDF}_{3}$ evaluation is negative. With the increase of the nodule radius, the accuracy of the $\mathrm{CDF}_{3}$ mapping by the chord method increases and for the particles with big sizes, the accuracy of mapping is reasonable.

It was assumed that the spherical particles of graphite with a radii smaller than $6 \mu \mathrm{m}$ are not present in the samples. Dependences of cumulative amount of the nodular graphite particles, whose radius does not exceed a given value, for samples No. I and II are shown in the Fig. $5 \mathrm{~b}$.

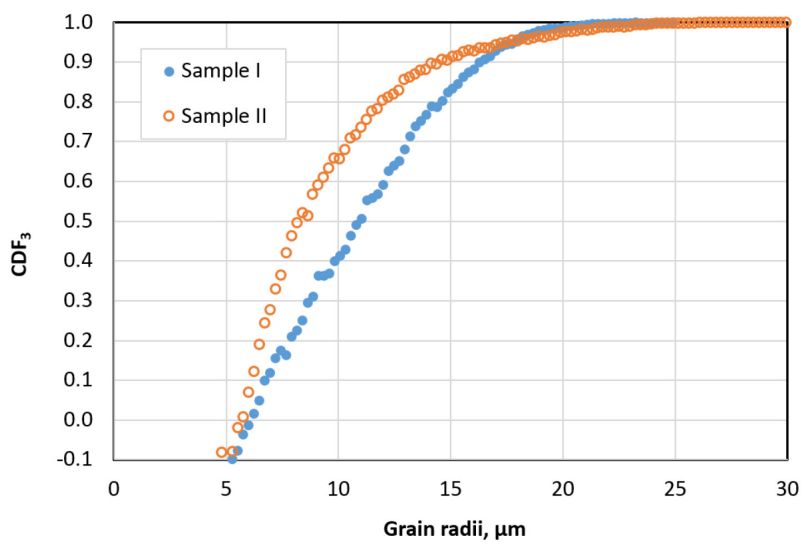

a)

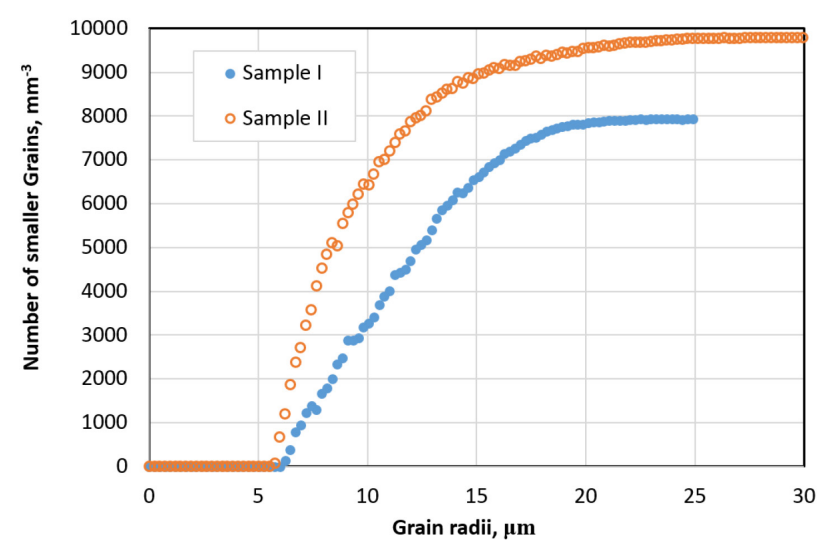

b)

Fig. 5. Cumulative distribution function of grain nodules in the sample I and II obtained by Eq. 2 (a) and volumetric cumulative number of the spherical graphite particles (b).

\section{Summary}

Empirical form of the cumulative distribution function of the sizes of graphite nodules in the DI can be mapped on the basis of the empirical measurement of probability density function of the statistical distribution of the length of random chords of the nodules by Eq. (2). Results of the experimental data processing are presented for two samples of industrial DI.

The chord method has a low accuracy at the interval nearby to $10 \%$ of the smallest spheroids of the analyzed population (like in the case of virtual one [17]). With the increase of the nodule radius, the accuracy of the mapping of the cumulative distribution function of the graphite nodules by the chord method increases and for the particles with big sizes, the accuracy of mapping is reasonable. 


\section{Acknowledgment}

The presented study was financed by the Polish Ministry of Science and Higher Education. Project AGH No 11.11.170.318 (8).

\section{References}

[1] Y. Yin, Z. Tu, J. Zhou, D. Zhang, M. Wang, Z. Guo, C. Liu, X. Chen, 3D Quantitative Analysis of Graphite Morphology in Ductile Cast Iron by X-ray Microtomography, Metall. and Mater. Trans. A, 48 (2017) 3794-3803

[2] R.E. Ruxanda, D.M. Stefanescu, T.S. Piwonka, Microstructure Characterization of Ductile Thin Wall Iron Castings, AFS Trans., 2 (2002) 1131-1148.

[3] K. Wiencek, T. Skowronek, B. Khatemi, Graphite Particle Size Distribution in Nodular Cast Iron, Metallurgy and Foundry Eng., 31 (2005) 167-174.

[4] S.D. Wicksell, The Corpuscle Problem: A Mathematical Study of a Biometric Problem. Biometrika, 17 (No. 1/2) (1925), 84-99.

[5] E. Sheil: Statistische Gefügeuntersuchungen I. Z. Metallk., 27 (1935) 199-208.

[6] H.A. Schwartz: The Metallographie Determination of the Size Distribution of Temper Carbon Nodules, Metals and Alloys, 5 (1934) 139-140.

[7] S.A. Saltykov: Stereometric Metallurgy, Metallurgizdat, Moscow, 1952.

[8] S.A. Saltykov: The determination of the size distribution of particles in an opaque material from the measurement of the size distribution of their sections. Stereology. Berlin, Heidelberg, Springer, 1967, 163-173.

[9] T. Li, S.-I. Shimasaki, Sh. Taniguchi, Sh. Narita: Reliability of Inclusion Statistics in Steel Stereological Methods. ISIJ International, 56 (No. 9) (2016) 1625-1633.

[10] M. Kong, R.N. Bhattacharya, C. James, A. Basu: A statistical approach to estimate the 3D size distribution of spheres from 2D size distributions. GSA Bulletin, 117 (No. 1/2) (2005), 244-249.

[11]A.J. Jakeman, R.S. Anderssen: Abel type integral equations in stereology. I. General discussion, Journal of Microscopy. 105 (1975), 121-133.

[12]J. Ohser, K. Sandau: Considerations About the Estimation of the Size Distribution in Wicksell's Corpuscle Problem, Lecture Notes in Physics, 554 (2000) 185-202.

[13]A. Burbelko, D. Gurgul, T. Wiktor: Stereological Analysis of Spherical Particles Size Distribution - Theoretical Basis, Accepted for print in Archives of Foundry Engineering, 18 (2018).

[14]J. W. Cahn, R. L. Fullman: On the Use of Lineal Analysis for Obtaining Particle-Size Distribution Functions in Opaque Samples, Trans. AIME, J. Metals, 206 (1956) 610-612.

[15] C.W. Lord, T.F. Willis: Calculation of air bubble distribution from results of a Rosiwal traverse of aerated concrete, A.S.T.M. Bull., 177 (1951) 177-187.

[16]A.G. Spektor: Analysis of distribution of spherical particles in non-transparent structures, Zavodsk. Lab., 16 (1950) 173-177.

[17]A. Burbelko, D. Gurgul, T. Wiktor: Stereological Analysis of Spherical Particles Size Distribution - Validation, Archives of Foundry Engineering, 17 (2017), 67-72. 